\title{
The optical detection unit for Baikal-GVD neutrino telescope
}

\author{
A.D. Avrorin ${ }^{1}$, A.V. Avrorin ${ }^{1}$, V.M. Aynutdinov ${ }^{1, a}$, R. Bannash ${ }^{7}$, I.A. Belolaptikov², \\ D.Yu. Bogorodsky ${ }^{3}$, V.B. Brudanin ${ }^{2}$, N.M. Budnev ${ }^{3}$, I.A. Danilchenko ${ }^{1}$, G.V. Domogatsky ${ }^{1}$, \\ A.A. Doroshenko ${ }^{1}$, A.N. Dyachok ${ }^{3}$, Zh.-A.M. Dzhilkibaev ${ }^{1}$, S.V. Fialkovsky ${ }^{5}$, \\ A.R. Gafarov ${ }^{3}$, O.N. Gaponenko ${ }^{1}$, K.V. Golubkov ${ }^{1}$, T.I. Gress ${ }^{3}$, Z. Honz ${ }^{2}$, K.G. Kebkal ${ }^{7}$, \\ O.G. Kebkal ${ }^{7}$, K.V. Konischev², E.N. Konstantinov ${ }^{3}$, A.V. Korobchenko ${ }^{3}$, A.P. Koshechkin ${ }^{1}$, \\ F.K. Koshel ${ }^{1}$, A.V. Kozhin ${ }^{4}$, V.F. Kulepov ${ }^{5}$, D.A. Kuleshov ${ }^{1}$, V.I. Ljashuk ${ }^{1}$, M.B. Milenin ${ }^{5}$, \\ R.A. Mirgazov ${ }^{3}$, E.R. Osipova ${ }^{4}$, A.I. Panfilov ${ }^{1}$, L.V. Pan'kov' ${ }^{3}$, A.A. Perevalov ${ }^{3}$, \\ E.N. Pliskovsky², M.I. Rozanov ${ }^{6}$, V.Yu. Rubtzov ${ }^{3}$, E.V. Rjabov ${ }^{3}$, B.A. Shaybonov' ${ }^{2}$, \\ A.A. Sheifler ${ }^{1}$, A.V. Skurihin ${ }^{4}$, A.A. Smagina ${ }^{2}$, O.V. Suvorova ${ }^{1}$, V.A. Tabolenko ${ }^{3}$, \\ B.A. Tarashansky ${ }^{3}$, S.A. Yakovlev ${ }^{7}$, A.V. Zagorodnikov ${ }^{3}$, V.A. Zhukov ${ }^{1}$, and V.L. Zurbanov ${ }^{3}$ \\ ${ }^{1}$ Institute for Nuclear Research, 60th October Anniversary pr. 7A, Moscow 117312, Russia \\ 2 Joint Institute for Nuclear Research, Dubna 141980, Russia \\ ${ }^{3}$ Irkutsk State University, Irkutsk 664003, Russia \\ ${ }^{4}$ Skobeltsyn Institute of Nuclear Physics MSU, Moscow 119991, Russia \\ ${ }^{5}$ Nizhni Novgorod State Technical University, Nizhni Novgorod 603950, Russia \\ ${ }^{6}$ St. Petersburg State Marine University, St. Petersburg 190008, Russia \\ ${ }^{7}$ EvoLogics GmbH, Berlin, Germany
}

\begin{abstract}
The first stage of the GVD-cluster composed of five strings was deployed in April 2014. Each string consists of two sections with 12 optical modules per section. A section is the basic detection unit of the Baikal neutrino telescope. We will describe the section design, review its basic elements - optical modules, FADC readout units, slow control and calibration systems, and present selected results for section in-situ tests in Lake Baikal.
\end{abstract}

\section{Introduction}

The objective of the Baikal Project is the creation of a kilometer-scale high-energy neutrino observatory: the Gigaton Volume Detector (GVD) in Lake Baikal [1-3]. The first phase of GVD will consist of 12 clusters of strings - functionally independent subarrays connected to the shore by individual electro-optical cables. Each cluster comprises eight strings of optical modules - seven peripheral strings are uniformly arranged at $60 \mathrm{~m}$ distance around a central one. Each string minimally comprises $24 \mathrm{OMs}$. The possibility of installing $36 \mathrm{OM}$ strings is to be studied at 2015-2016.

The present configuration of the first GVD-cluster compris five strings and was deployed in 2014. within the framework of BAIKAL-GVD project. Each string consists of two sections with 12 optical modules each. A section is the basic detection unit of the Baikal neutrino telescope. We describe the section design, review the basic section elements - optical

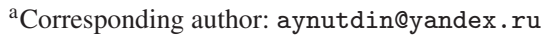

(C) The Authors, published by EDP Sciences. This is an Open Access article distributed under the terms of the Creative Commons Attribution License 4.0 (http://creativecommons.org/licenses/by/4.0/). 

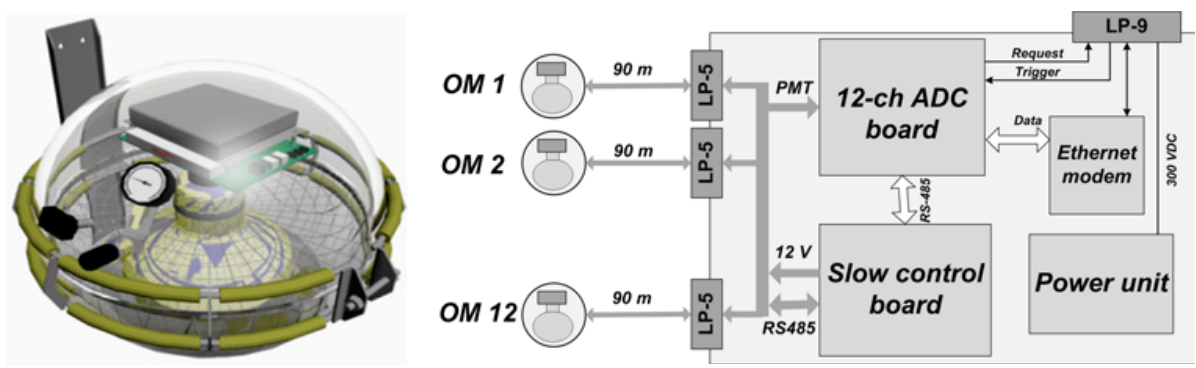

Figure 1. A sketch of a GVD optical module and a block diagram of the GVD section.

modules, FADC readout units, slow control and calibration systems and present selected results of section element in-situ testing in Lake Baikal.

\section{GVD section}

A section is the basic detection unit of the GVD neutrino telescope [4]. It comprises 12 optical modules $(\mathrm{OM})$ and a central module $(\mathrm{CeM})$. The block diagram of a section is presented in Fig. 1. PMT signals from all OMs are transmitted to the CeM via 90 meters of coaxial cables, where they are digitized by custom-made 12-channel ADC boards with $200 \mathrm{MHz}$ sampling rate. The slow-control board located in the CeM provides data communication between OM and $\mathrm{CeM}$ via an underwater RS-485 bus. Also, this unit is intended for OM power control (to switch power on/off for each optical module independently). The ADC board provides trigger logic, data readout and digital processing, and connection via local Ethernet to the cluster DAQ center.

$\mathrm{OM}$ and $\mathrm{CeM}$ electronics are placed in a pressure-resistant glass sphere VETROVEX with $42 \mathrm{~cm}$ diameter. One connector SubConn Low Profile 5-contacts is installed on each optical module. It is used for analog pulse transmission, slow control (2-wire RS-485) and OM power supply (12 VDC). CeM has twelve 5-contact connectors to connect with OMs and one 9-contact SubConn connector for communication with the DAQ centre of the cluster.

\section{Optical module}

The optical modules [5] contain the photo-sensors of GVD: a photomultiplier tube (PMT), which detects the Cherenkov light produced by relativistic charged particles passing through the water. After testing different options, the photomultiplier Hamamatsu R7081-100 was selected as a light sensor for the OM. This PMT has a hemispherical photocathode with 10 inch diameter and a typical quantum efficiency of $35 \%$. The PMT is placed in a pressureresistant glass sphere. A high permittivity alloy cage surrounds the PMT, shielding it against the Earth's magnetic field. The OM electronics unit is mounted directly onto the PMT base. The OM electronics includes a controller, a high voltage (HV) power supply unit, a fast twochannel amplifier, and a LED flasher. The block diagram and a picture of the optical module electronics are presented in Fig. 2.

The OM controller is intended for HV regulation and monitoring, for PMT noise measurements, and for time and amplitude calibration. It is designed on the basis of SiLabs C8051F121 microcontroller. Slow control data to and from the OMs are transferred via an underwater RS-485 bus. The unit has an instruction set for the control of OM electronics: HV control (to switch HV on/off, to set PMT voltage and readout HV value), LED flasher 


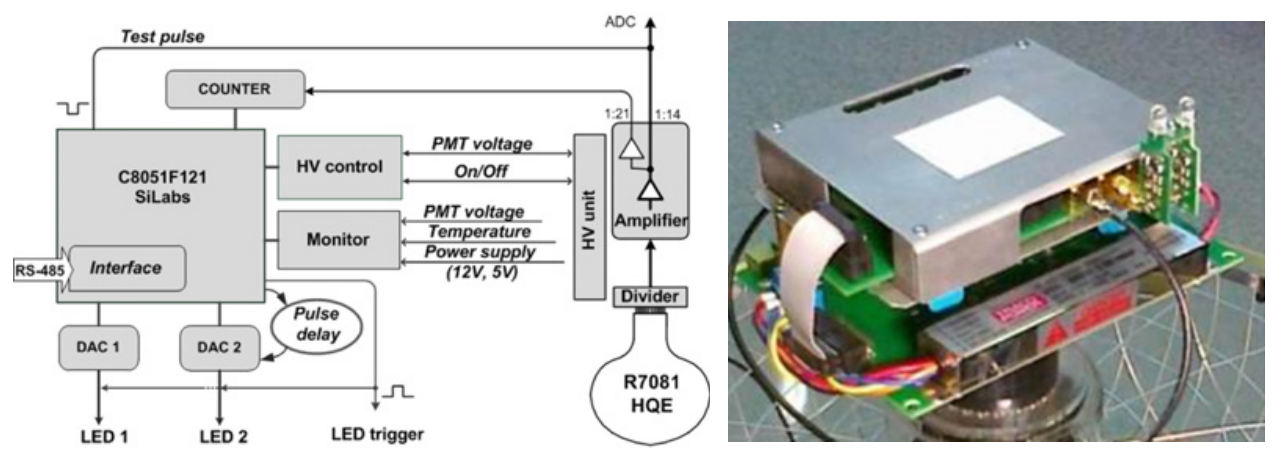

Figure 2. Block diagram and a picture of the OM electronics.
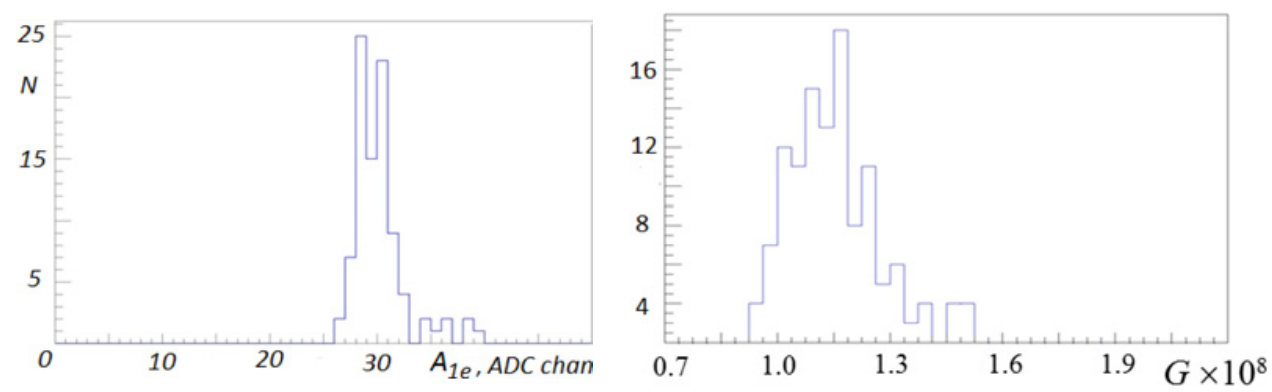

Figure 3. Distributions of the single electron amplitude $A_{1 e}$ and the gains $G$ (12 May 2014).

control (to set LED intensities, the delay between LED pulses, and the period of flashes), the counter control (setting counter threshold, time window, off-duty factor and the size of circular memory data buffer). Also a set of procedures is foreseen for the laboratory calibration of the counter threshold and PMT voltage.

PMT power supply is provided by a passive HV divider circuit with the resistance of 18 $\mathrm{M} \Omega$ and HV unit Traco Power SHV 12-2.0 K $1000 \mathrm{P}$ with positive polarity. The tube gains have been adjusted to about $10^{7}$. This gain is provided by divider voltages between $1150 \mathrm{~V}$ and $1800 \mathrm{~V}$, depending on the individual tube (average value is about $1400 \mathrm{~V}$ ). An additional signal amplification is provided by the amplifier. The PMT amplifier is comprised of two channels. The first channel with an amplification factor of 14 forms a signal with negative polarity that is transmitted to the ADC board. The second channel with an amplification factor of 21 produces positive pulses that are intended for PMT noise monitoring. The outer cascades are implemented as emitter-follower amplifiers and provide operation with $50 \Omega$ load. The maximum amplitude for both channels is limited to 4 Volts. Assuming an attenuation factor of 0.7 over 90 meter coaxial cable, the total channel amplification will be about $10^{8}$. This corresponds to a single photoelectron value at 30 ADC channels (ADC scale is $1.25 \mathrm{mV}$ per channel). During detector operation in 2014 several special runs were conducted to check the PMT gains. Distributions of single photoelectron amplitudes and the channel gains for May 2014 are presented in Fig. 3 (the average gain is $1.12 \times 10^{8}$ ). The same measurements conducted in July and August have shown a stability of the average channel gain: $1.17 \times 10^{8}$ and $1.14 \times 10^{8}$, respectively.

The LED flasher is intended for time and amplitude calibration of OM channels during long-term exposition. It includes two LEDs Kingbright L7113 and electronic circuits: LED 


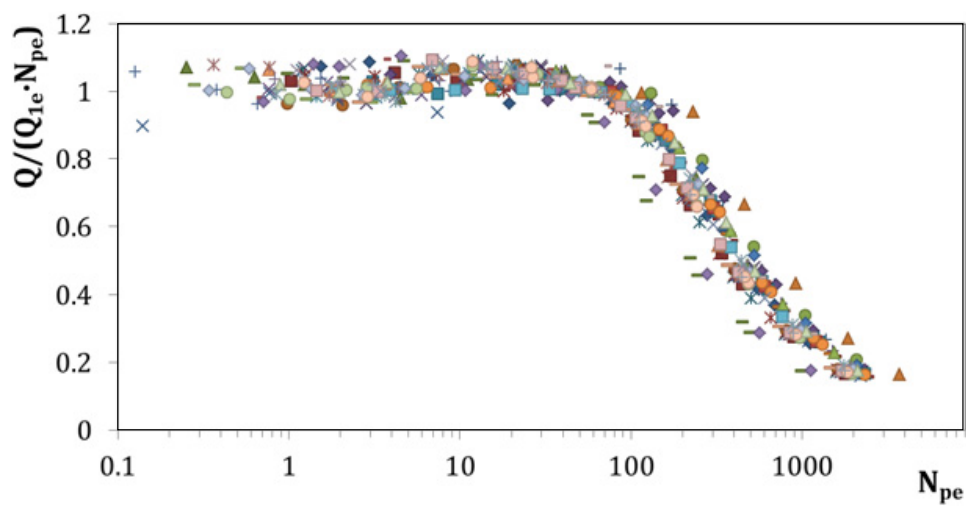

Figure 4. The channel nonlinearity curves (marked by symbols) for $24 \mathrm{OM}$ as a function of photoelectron numbers $N_{\text {pe }}$.

drivers. The dominant wavelength of the LED is $470 \mathrm{~nm}$, the LED pulse has a width of $\sim 6 \mathrm{~ns}$ (FWHM). The control system of the flasher has to provide independent smooth regulation of two LED luminosities in a wide dynamic range (up to about $10^{8}$ photons per LED flash), has to have minimal level of cross-talks between two LED channels (less than 1\%). Independent regulation of light intensities of each flasher channel and absence of cross-talks provide a possibility to perform a linearity test of spectrometric channels. Results of linearity test conducted in a laboratory for 24 optical modules (one string) are presented in Fig. 4 . The nonlinearity was calculated as $Q /\left(Q_{1 e} \times N_{p e}\right)$, where $Q$ is a measured PMT pulse charge, $Q_{1 e}$ is single electron charge, and $N_{p e}$ is a number of produced photoelectrons. The estimated linearity range is about 100 photoelectrons.

Another application of the LED flasher is a time calibration of the sections and channels. Time calibration means measurements of relative delays of signals from the individual channels. The channel delay accumulates two main components: cable delays and delays from the PMTs. Cable delays are measured once in the laboratory and do not change with time. PMT delays depend on power voltage and thus require regular calibration in situ. There is a specialized test pulse generated by the OM controller that is delivered to the point of signal creation in the PMT preamplifier. The difference between arrival times of the LED signal and the test pulse gives the delay of the PMT signal.

Due to the sufficiently large intensity of LED bursts, the signals generated by LEDs are detected by PMTs of neighboring sections and strings. The calibration coefficients are derived from the known positions of OMs (which are determined by acoustic methods). Figure 5 (left) shows an example of the detection of LED flashes with 8 OMs of one string. LED flasher on the first $\mathrm{OM}(\mathrm{R}=0)$ is switched on with a maximum light intensity. Each point on the plot shows the OM position on the string versus flashes detection time. The straight line corresponds to the velocity of light in the Baikal water. The average pulse amplitude for the most distant $\mathrm{OM}(\mathrm{R}=105 \mathrm{~m})$ is about 1 photoelectron.

The OM counter with programmable threshold is intended for PMT count rate monitoring. Count rate data is accumulated in the circular buffer and transmitted to the shore with 10 minute interval for each OM. Figure 5 (right) shows the count rate data for all channels accumulated from April to August 2014 for all OMs. The average channel rate is $23 \mathrm{kHz}$ for about 0.5 p.e. counter thresholds. 

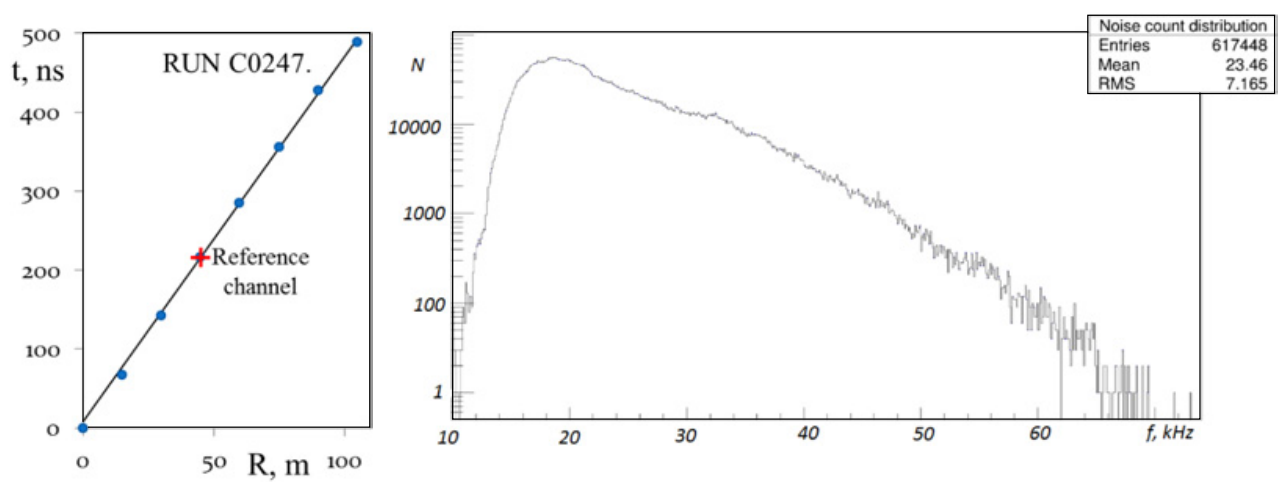

Figure 5. The PMT detection time of the LED flashes vs OM position on the string (left), and the count rates for all channels accumulated from April to August 2014 (right).
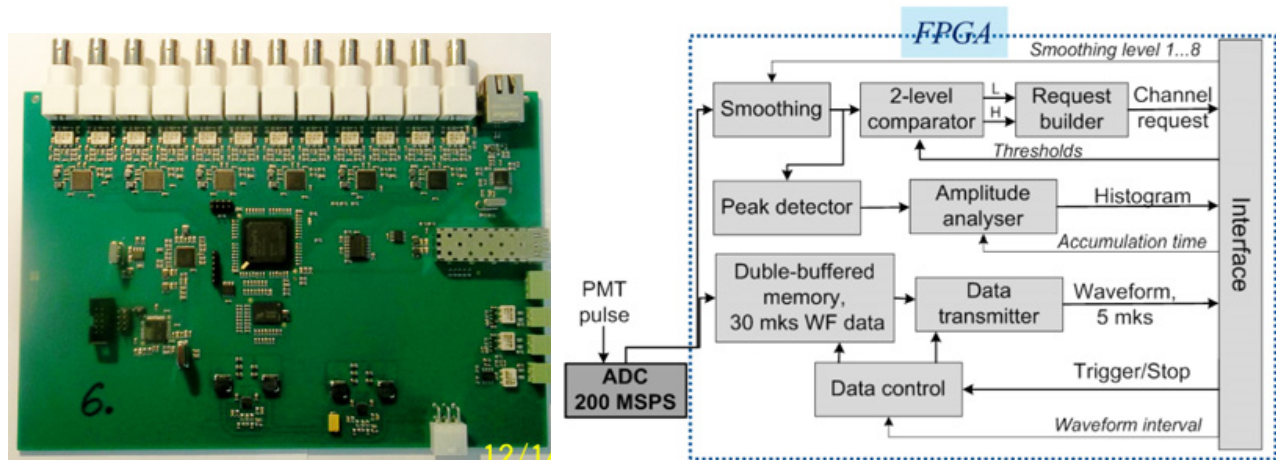

Figure 6. A picture of the custom made ADC board and a block diagram of the ADC channel.

\section{Central electronics module}

The basic elements of the central electronics module [6-9] are ADC board and slow control board (see Fig. 1). The ADC board is a 12-bit 200MS/s flash ADC waveform digitizer with 12 input channels. It provides trigger logic, data readout and connection via local Ethernet to the cluster DAQ center. The ADC board is implemented on the basis of microcircuits AD9613 and FPGA Xilinx Spartan 6. AD9613 is a dual 12-bit analog-to-digital converter with sampling speeds up to 250 MSPS (see Fig. 6). Data streams are continuously written into circular memory buffers of each ADC channel. When the trigger occurs, the digitizer freezes the buffer that can be read by readout links. The acquisition can continue without any dead time in the second buffer. A memory buffer of $12 \mathrm{kB}$ allows waveform data accumulation from the $\mathrm{ADC}$ for $30 \mu \mathrm{s}$. A common acquisition trigger signal can be provided externally as well as via the software, but it can also be generated internally thanks to self-trigger capability. Thanks to the FPGAs housed in the digitizer, ADC board provides possibilities of on-line signal processing rejecting samples smaller than programmable threshold. It allows for reducing the data flow more than 50 times.

Each ADC data channel is connected to a peak detector and an amplitude analyzer, which accumulates monitor histograms in a programmable time interval. An ADC trigger channel includes a smoothing unit for electronics noise reduction, a two-level adjustable digital comparator (low threshold $\mathrm{L}$ and high threshold $\mathrm{H}$ ), and a request builder, that builds the 
trigger logic request. The basic trigger modes are (A) coincidences of $>\mathrm{N}$ L-requests within a selectable time window or (B) coincidences of $\mathrm{L}$ and $\mathrm{H}$ requests from any neighbouring $\mathrm{OMs}$ within a section.

The section data is transferred through Ethernet channel. Two Ethernet outputs are foreseen: DSL-modem (MOXA IEX-402-SHDSL) and optical output on ADC board for future detector extension.

\section{Conclusion}

The present configuration of the first GVD-cluster consists of five strings and has been deployed in 2014. The basic detection units of GVD are sections of optical modules (OM), with each OM housing a photomultiplier of the type R7081-100. In-situ tests of the installation in Lake Baikal show good performance of all section elements. The prototyping phase will be concluded in 2015 with the completion of the first GVD demonstration cluster with 8 strings.

This work was supported by the Russian Found for Basic Research (grants 13-02-12221, 14-02-00175, 14-02-00972).

\section{References}

[1] A. Avrorin, et al., Astronomy Letters 37, 7 (2011)

[2] A. Avrorin et al., Nucl. Instr. and Meth. A626, 6 (2011)

[3] A. Avrorin et al., Nucl. Instr. and Meth. A630, 4 (2011)

[4] A. Avrorin et al., Nucl. Instr. and Meth. A639, 3 (2011)

[5] A. Avrorin et al., Instruments and Experimental Techniques 54, 649-659 (2011)

[6] A. Avrorin et al., Nucl. Instr. and Meth. A692, 7 (2012)

[7] A. Avrorin et al., Nucl. Instr. and Meth. A693, 10 (2012)

[8] A. Avrorin et al., Nucl. Instr. and Meth. A725, 4 (2013)

[9] A. Avrorin et al., Instruments and Experimental Techniques 3, 12 (2014) 